\title{
ZWISCHEN WEISHEIT UND WISSENSCHAFT SCHLICKS WEITES PHILOSOPHISCHES SPEKTRUM ${ }^{1}$
}

\author{
Thomas MORMANN \\ University of the Basque Country UPV/EHU, Spain
}

Moritz Schlick, oft als Haupt des Wiener Kreises apostrophiert, ist in der philosophischen Diskussion heute weniger präsent als manches „einfache Mitglied“ des Kreises oder als viele seiner Zeitgenossen, die irgendwann als „dem Kreis Nahestehende“, oder „zur Peripherie Gehörende“ mit dem Kreis in mehr oder minder enger Beziehung standen - man denke an Carnap, Neurath, Popper, Gödel, Tarski, Russell oder Wittgenstein. Die Gründe für Schlicks relative Unbekanntheit sind vielfältig. Einer ist sicher sein früher Tod, der verhinderte, dass sein Werk in der angelsächsischen Philosophie bekannt wurde, und deshalb auch nicht unter dem attraktiven Rubrum ,analytische Philosophie“ in die deutsche Philosophie reimportiert werden konnte. Ein anderer Grund könnte darin liegen, dass es überhaupt schwierig ist, für Schlicks Denken einen passenden Ort in der Landschaft der Philosophie des 20. Jahrhunderts zu finden. Weder seine philosophischen Interessen noch sein philosophischer Stil erlauben es, ihn umstandslos als logischen Empiristen zu charakterisieren, aber auch andere Etiketten wie Proto-empirizist, Empiriokritizist, kritischer Realist oder dergleichen wollen nicht recht passen oder erweisen sich als wenig hilfreich. Man sollte ihn deshalb ohne klassifikatorische Scheuklappen lesen und dabei auf einige Überraschungen gefasst sein - Schlick war kein logischer Empirist, wie er im Buche steht.

1. Besprechungsaufsatz zu folgenden Neuerscheinungen:

Mathias Iven, Moritz Schlick. Die frühen Jahre (1882-1907). Berlin: Parerga, 2008 (Schlickiana Band 2) 238 Seiten. ISBN 13 978-3-937-26284-0.

Moritz Schlick, Kritische Gesamtausgabe. Herausgegeben von Friedrich Stadler und HansJürgen Wendel, Wien und New York: Springer, 2006ff.

Abteilung I, Band 2: Über die Reflexion des Lichtes in einer inhomogenen Schicht und Raum und Zeit in der gegenwärtigen Physik. Herausgegeben von Fynn Ole Engler und Mathias Neuber, 2006, 412 Seiten. ISBN 13 978- 3-211-29785-8. (MSGA 2)

Abteilung I, Band 3: Lebensweisheit, Versuch einer Glückseligkeitslehre und Fragen der Ethik. Herausgegeben von Matthias Iven, 2006, 581 Seiten. ISBN 13 798-3-211-29789-6 (MSGA 3).

Abteilung I, Band 6: Die Wiener Zeit. Aufätze, Beiträge, Rezensionen 1926-1936. Herausgegeben von Johannes Friedl und Heiner Rutte, 2008, 946 Seiten. ISBN 13 978-3-211-33114-9.

(MSGA 6) 
Die Möglichkeiten einer ernsthaften und umfassenden Rezeption Schlicks haben sich in den letzten Jahren erheblich verbessert. Unter der Gesamtherausgeberschaft von Friedrich Stadler und Hans Jürgen Wendel sind seit 2006 von der auf etwa fünfzehn Bände angelegten „Moritz Schlick Gesamtausgabe“ (MSGA) vier Bände erschienen. Auch die Sekundärliteratur ist im Anschwellen begriffen, und die ersten Bände der auf die Erforschung von Schlicks Philosophie spezialisierten Zeitschriften Schlickiana und Schlickstudien sind erschienen. Die bisher vorliegenden Bände der Gesamtausgabe (MSGA) sind editorisch hervorragend gestaltet, jeder Klassiker, salopp ausgedrückt, ist postum zu beglückwünschen, wenn er mit einer solchen Ausgabe seiner Werke bedacht wird. Das gilt nicht nur für die Ausstattung der Bände selbst, die auch gehobenen bibliophilen Ansprüchen genügt, es gilt insbesondere für die sorgfältigen und ausführlichen Einleitungen und Kommentare der Herausgeber.

Wie wenig der "ganze“ Schlick im Bewußtsein der allgemeinen philosophischen Öffentlichkeit verankert ist, belegt ein 1996 in den Wittgenstein Studien erschienener Bericht über ein Gedenksymposium, welches die Universität Wien aus Anlaß von Schlicks 60. Todestag abgehalten hatte und in dem schlicht vom „österreichischen Philosophen“ Moritz Schlick die Rede ist. Dieser Lapsus spiegelt prägnant die lange Zeit vorherrschende Rezeption Schlicks wider, die ihn auf seine Rolle im Wiener Kreis reduzierte und seine philosophische vor-wiener Vergangenheit vollständig ausblendete. Das ist ein Fehler, denn es wäre keineswegs völlig abwegig, die Allgemeine Erkenntnislehre (1918) als Schlicks wichtigstes Werk anzusehen, das er verfasste, geraume Zeit bevor er nach Wien ging, und von dem er sich überdies in Wien mehr und mehr distanzierte.

Lange Zeit war über den frühen Schlick, seine Lebensumstände und seine wissenschaftlichen und philosophischen Ursprünge recht wenig bekannt. Hier füllt Mathias Ivens Moritz Schlick. Die frühen Jahre (1882 - 1907) eine empfindliche Lücke. Iven konzentriert sich auf die Erforschung von Schlicks äußeren Lebensumständen. Da sich diese als keineswegs bedeutungslos für die Inhalte seiner Philosophie erweisen, kann sein Buch auch als eine „intellektuelle Biographie“ des jungen Schlick gelesen werden.

Schlicks philosophische Anfänge liegen in einer Zeit, die man grob als die wilhelminische Epoche auf dem Höhepunkt ihres äußeren Glanzes beschreiben kann. Die „philosophische Gestalt“ dieser Zeit ist uns heute jedoch noch keineswegs klar, sie war bestimmt durch zahlreiche, oft antagonistische Einflüsse wie die verschiedenen Strömungen der Lebensphilosophie, einen weit verbreiteten Nietzscheanismus, die neuromantische Zivilisationskritik der Jugendbewegung, und in der akademischen Sphäre durch die Philosophie des Neukantianismus, um nur einige zu nennen. 
Geboren 1882 in einem großbürgerlichen, wohlhabenden Elternhaus, das nicht ungern die nach Ivens Recherchen allerdings zweifelhaften aristokratischen Wurzeln der Familie betonte (cf. Iven 2008, 15ff), wuchs Moritz Schlick als jüngster Sohn von Albert Schlick und seiner Frau Wilhelmine, geb. Arndt, im Berlin des Zweiten Kaiserreiches auf. Der Tätigkeitsbereich des väterlichen Unternehmens, eine „Elfenbeinhandlung und Dampfschneide-Anstalt“, erinnert eher an eine Manufaktur des frühen 19. Jahrhunderts als an ein modernes industrielles Unternehmen, scheint aber zumindest bis zum Beginn des ersten Weltkriegs so viel Profit abgeworfen zu haben, dass der Vater seinem Sohn Moritz und dessen junger Familie ein materiell sorgenfreies Leben ermöglichen konnte. Der junge Schlick führte das Leben eines wissenschaftlich begabten jungen Mannes aus wohlsituiertem Hause, der für seinen Lebensunterhalt nicht selber aufkommen musste, zunächst Physik studierte, in diesem Fach auch promovierte, aber eigentlich noch nicht genau wußte, was er machen wollte. Für längere Zeit schwankte Schlick zwischen Physik, Psychologie und Philosophie und widmete sich außerdem noch intensiv seinem philosophischen Hobby, nämlich der Ausarbeitung seines ersten Buches Lebensweisheit. Versuch einer Glückseligkeitslehre. Bereits während seiner Gymnasialzeit hatte er sich mit dieser Thematik beschäftigt, veröffentlicht wurde das Buch schließlich 1908. Iven hat in Die frühen Jahre minutiös die aus dieser Periode noch vorhandenen spärlichen Daten zusammengetragen, so dass wir jetzt ein einigermaßen vollständiges Bild von Schlicks Lebensumständen, seinen Plänen und Projekten aus dieser Zeit haben. Einige immer noch vorhandene Lücken werden sich aber wohl nicht mehr schließen lassen.

Nach dem Abitur begann Schlick im Wintersemester 1900 mit dem Studium der Physik an der Friedrich-Wilhelms-Universität zu Berlin. Nach Gastsemestern in Heidelberg und Lausanne kehrte er nach Berlin zurück und begann im Wintersemester 1903/04 mit einer Dissertation aus dem Gebiet der klassischen Strahlenoptik unter der Leitung Max Plancks. Die Arbeit Über die Reflexion des Lichtes in einer inhomogenen Schicht erhielt das Prädikat „cum laude“ und im Mai 1904 wurde Schlick mit „magna cum laude“ promoviert. Obwohl Schlick nach seiner Dissertation in der Physik keine nennenswerte Arbeit mehr veröffentlichte, blieb er Zeit seines Lebens mit seinem Doktorvater Planck in freundschaftlichem Kontakt. Noch 1938 erinnert sich Planck seiner als eines seiner bemerkenswertesten Schüler. Umgekehrt blieben Plancks wissenschaftliche und philosophische Überzeugungen für Schlick ein Orientierungspunkt, den er bei seinen eigenen philosophischen Explorationen nicht aus den Augen verlor, auch wenn er, besonders in seiner Wiener Zeit, mit Planck keineswegs immer einer Meinung war. Die genauere Erforschung der Beziehungen zwischen Schlick und seinem wissenschaftlichen Mentor Planck dürfte in den nächsten Jahren ein wichtiges Thema der Schlick-Forschung bilden. 
Bis zur Promotion kann Schlicks Lebenslauf als Musterbeispiel für den Beginn einer erfolgreichen wissenschaftlichen Laufbahn gelten: Immatrikulation an einer renommierten Universität, zügiges Studium ohne Umwege, und eine erfolgreiche Promotion bei einer Koryphäe seines Faches versprachen für die Zukunft eine glänzende wissenschaftliche Laufbahn. Diese gradlinige Entwicklung setzte sich nach der Promotion zunächst jedoch nicht fort. Es begann eine Phase, die man nicht nur im geographischen Sinne als Schlicks Wanderjahre bezeichnen kann, und die ihn auf recht verschlungenen Pfaden von der Physik schließlich zur Philosophie führten. Es ist ein Verdienst von Ivens Die frühen Jahre, die Geschehnisse dieser Zeit minutiös rekonstruiert zu haben.

Schlicks Dissertation Über die Reflexion des Lichtes in einer inhomogenen Schicht (1904) (MSGA 2) war seine erste Publikation überhaupt und zugleich auch seine letzte nennenswerte Veröffentlichung in der Physik. Obwohl Planck ein höchst angesehener Physiker war, hatte er nur wenige Doktoranden. Er galt als „schwierig“, weil er von seinen Schülern große Selbständigkeit verlangte. Bei Planck zu promovieren, war deshalb etwas Besonderes. Schlicks Dissertation war eine theoretische Arbeit aus dem Bereich der Optik im Rahmen von Maxwells elektromagnetischer Theorie des Lichtes. Es ging um das Verhalten von Lichtstrahlen in inhomogenen Medien, genauer um die Frage, ob „beim Durchgange des Lichtes durch ein Medium mit räumlich variablem Brechungsexponenten stets Reflexionen auftreten, oder ob solche nur eintreten, wenn die optischen Eigenschaften des Mittels im Raume unstetig sind." (MSGA 2, 63) Schlick beantwortete diese Frage für bestimmte Fälle positiv, indem er die zugehörigen Maxwellschen Differentialgleichungen explizit aufstellte und integrierte. Er wies jedoch darauf hin, dass eine empirische Bestätigung seiner Resultate noch ausstehe. Im wissenschaftsphilosophischen Vokabular Kuhns ist Schlicks Dissertation wohl ein typischer Fall von Normalwissenschaft, in dem ein allgemeines, nicht in Frage gestelltes Paradigma auf einen speziellen Fall erfolgreich angewendet wird, wodurch der „Novize“ unter Beweis stellt, dass er die paradigmaspezifischen Methoden seiner Wissenschaft anzuwenden weiss, um ein „Puzzle“ zu lösen.

Nach der Promotion immatrikulierte Schlick sich zunächst wieder in Göttingen, um sich mit Experimentalphysik zu befassen, gleichzeitig setzte er die bereits 1902 begonnene Arbeit an einem Manuskript fort, aus dem schließlich sein erstes Buch Lebensweisheit hervorgehen sollte. Die Arbeit im Göttinger Labor für Experimentalphysik befriedigte ihn jedoch in keiner Weise. Er verbrachte viel Zeit mit Reisen und Kuraufenthalten. So war er Anfang 1905 einige Wochen in Heidelberg „um der schönen Landschaft willen“ (Schlick nach Iven, 111). Dort lernte er seine spätere Frau Blanche Guy Hardy kennen, die aus einer in Massachusetts ansässigen methodistischen Pastorenfamilie stammte. In den fol- 
genden Monaten arbeitete er weiter an der Lebensweisheit, sprach mit Eucken über die Möglichkeit, in Jena als Philosoph zu arbeiten, gab diesen Plan aber auf, da er mit dessen philosophischer Orientierung nichts anfangen konnte, und ging dann für einige Semester (bis Anfang 1910) nach Zürich, um Vorlesungen in Psychologie bei Gustav Störring zu hören (cf. Iven 2008, 167ff).

Im September 1907 reist er nach Massachusetts, um dort seine Verlobte Blanche zu heiraten. Nach ihrer Rückkehr nach Europa ließ sich das junge Paar in Zürich nieder. Mitte 1909 stellte Schlick den Antrag auf Zulassung als Privatdozent an der Universität Zürich, sein Antrag wurde aber abgelehnt, da er zu dieser Zeit noch kaum etwas veröffentlicht hatte. Auch Pläne für eine Habilitation in Kiel und Gießen zerschlugen sich, so dass Schlick mit Frau und Sohn zunächst zu seinen Eltern nach Berlin zurückkehrte. Im Sommer 1910 zog er mit seiner Familie nach Rostock, wo er im Mai 1911 mit der Habilitationsschrift Das Wesen der Wahrheit nach der modernen Logik schließlich die venia legendi im Fach Philosophie erlangte.

Nichts an diesem etwas krausen Curriculum des jungen Privatdozenten deutete darauf hin, dass dieser kaum zehn Jahre später zum institutionellen Mittelpunkt eines der wichtigsten Zentren der wissenschaftlichen Philosophie in Europa avancieren würde. Zwar konnte seine „gediegene Dissertation“ (Planck) als Ausweis solider Kenntnisse in Physik gelten, Wissenschaftsphilosophie im eigentlichen Sinne aber war noch keineswegs der ausgewiesene Schwerpunkt von Schlicks philosophischer Arbeit. Außer der Habilitationsschrift hatte er nur den Aufsatz Das Problem der Ästhetik in entwicklungsgeschichtlicher Hinsicht (1909) veröffentlicht. Beide Arbeiten hatten wenig oder nichts mit Wissenschaftsphilosophie der empirischen Wissenschaften zu tun. Der Schwerpunkt seiner philosophischen Arbeit lag damals noch eindeutig auf der Ethik. Das wird noch deutlicher, wenn man nicht nur seine veröffentlichten Schriften wie Lebensweisheit berücksichtigt, sondern auch die kurz nach der Fertigstellung von Lebensweisheit begonnenen (und später aufgegebenen) Werke Der neue Epikur und Die Philosophie der Jugend.

Motive und Stilelemente der Lebensweisheit lassen sich in Schlicks gesamtem Philosophieren finden, so dass man dieses Werk durchaus nicht als eine jugendliche Verirrung abtun sollte, die nichts mit seinem „eigentlichen“ Werk zu tun hätte. Lebensweisheit ist ein Dokument, das viel zum Verständnis von Schlicks späterem Denken beiträgt und die intellektuellen Ausgangspunkte seiner Entwicklung sichtbar werden lässt, von denen er sich - entgegen dem Anschein - niemals sehr weit entfernt hat. In den folgenden Erörterungen behandeln wir daher zunächst Lebensweisheit und Fragen der Ethik (MSGA 3), gehen dann ein auf Raum und Zeit in der gegenwärtigen Physik (MSGA 2) und betrachten schließlich die in Die Wiener Zeit. Aufsätze, Beiträge, Rezen- 
sionen 1926-1936 (MSGA 6) versammelten Arbeiten aus seinem letzten Lebensjahrzehnt.

Auf den ersten Blick ist Lebensweisheit ein einigermaßen seltsames, bisweilen unfreiwillig komisches Buch. In ihm gehen das Streben nach Wissenschaftlichkeit und bildungsbürgerlicher Erbauung eine eigentümliche Mischung ein. Lebensweisheit kann als Versuch gelesen werden, auf „wissenschaftlicher“ Grundlage eine von bürgerlichen Idealen geprägte Utopie einer von allen Zwängen und Einschränkungen freien Gesellschaft zu formulieren, in der man nicht mehr zu arbeiten brauchte, in der der Gegensatz zwischen Kultur und Natur aufgehoben war, und wo die auf entfremdeter Arbeit beruhende Zivilisation der kapitalistischen Industriegesellschaft einer Kultur des „Spielerischen“ Platz gemacht hatte. In dieser Welt lebte zwar jeder sein Leben nach dem hedonistischen Prinzip der höchsten individuellen Lusterfüllung, diese bestand aber darin, seinen Beitrag zur Verwirklichung des Glücks der anderen zu leisten, was dann in einer allseitigen und universellen Harmonie aller Menschen mündete.

Lebensweisheit ist ein interessantes Dokument seiner Zeit, da Schlick in diesem Werk zahlreiche Ideen der damals populären philosophischen und (pseudo) wissenschaftlichen Strömungen verwendete, so z.B. von Nietzsche, Darwin und Stirner, garniert mit Versatzstücken aus dem klassischen Repertoire der bürgerlichen Kulturkritik wie den Gegensätzen von „Kultur und Zivilisation“, „Kultur und Natur“, „Körper und Seele“, der Unterscheidung von „höheren“ und „niederen“ Genüssen, der von der Industrialisierung verursachten Entfremdung und der Unterdrückung des natürlichen Trieblebens durch die Konventionen der bürgerlichen Gesellschaft usw.

Als idealen Gegenentwurf zur wilhelminischen Gesellschaft zu Anfang des 20. Jahrhunderts skizziert Schlick in Lebensweisheit ein Leben, das, etwas unfreundlich ausgedrückt, an eine Rokokoidylle des 18. Jahrhunderts erinnert. Dieser Entwurf ergibt sich für ihn aus seiner idiosynkratischen Interpretation der Darwinschen Evolutionstheorie, in die Momente einer von Nietzsche inspirierten Triebpsychologie eingehen. Danach ist das Handeln des Menschen grundsätzlich bestimmt vom „Willen zur Lust“ oder vom „Willen zum Glück“, was für Schlick auf dasselbe hinausläuft (MSGA 3, 94). Der Wille zum Glück ist das oberste und einzige Gesetz, dem der Mensch unbedingt gehorchen muß, nicht weil es ihm von außen auferlegt worden wäre, sondern weil es ihm immanent ist (MSGA 3, 108). „Lust“ entsteht aus der Befriedigung eines Triebes. Da es verschiedene, einander oft widerstreitende Triebe gibt, die nicht alle zugleich und sofort befriedigt werden können, stellt sich die Frage, wie diesem Imperativ des „Willens zur Lust“ am besten Folge zu leisten ist, welcher Trieb also derjenige ist, dessen Befriedigung die höchste Lust bereitet. Dieses Problem wird dadurch kompliziert, dass viele Triebe, zumindest in der real existierenden bürgerlichen 
Gesellschaft, nicht sofort befriedigt werden können. Freudianisch gesprochen, ist die bürgerliche Gesellschaft auf Triebaufschub und Triebverzicht aufgebaut. Konkret bedeutet das, man muß in dieser Gesellschaft arbeiten oder andere dazu bringen, für einen zu arbeiten, um danach (vielleicht) genießen zu können, also seinen Willen zum Glück befriedigen zu können.

Eine durch Arbeit erlangte Triebbefriedigung bleibt nach Schlick jedoch immer unvollkommen, einmal, weil jede Arbeit etwas Unnatürliches ist, das letzten Endes die Unterjochung und Beherrschung der Natur zum Zweck hat und so den Menschen in einen Gegensatz zur Natur bringt; zum anderen, weil Arbeit immer mit unlustvollen Handlungen verbunden ist, die nur der Vorbereitung der Triebbefriedigung dienen, selbst aber nicht lustvoll sind. Kurz, Arbeit schien dem jungen Gelehrten aus gutem Hause nicht der richtige Weg zur vollkommenen Glückseligkeit.

Den Inbegriff all dessen, was durch die Arbeit geschaffen worden ist, nennt Schlick Zivilisation. Die Zivilisation sei um des Glücks des Leibes willen erfunden worden, erlaube also höchstens die Befriedigung der leiblichen Triebe, die Schlick als minderwertig gegenüber den seelischen ansah. Entsprechend dem in Deutschland endemisch verbreiteten Topos von „Kultur versus Zivilisation“ definierte er Kultur im Gegensatz zur „Zivilisation“ als „Inbegriff aller künstlichen Mittel, die die Menschheit geschaffen hat, um ihre Glückseligkeit - des Leibes wie der Seele - zu mehren." (MSGA 3, 110) Aber nicht nur die Zivilisation „in ihrer gegenwärtigen Gestalt ... ist ... eine Verirrung“, auch „eine Kultur, welche Fabrik auf Fabrik baut, die Luft mit Lärm und Schmutz erfüllt, ... kann nur wenig Ähnlichkeit mit jener ... Kultur haben, wonach der Leib sich sehnt.“" (MSGA 3, 118)

Die technisch-industrielle Zivilisation ist also kein geeigneter Rahmen für die Verwirklichung des menschlichen Glücksstrebens. Gleichwohl werde, so Schlick optimistisch, die technisch-industrielle Zivilisation das menschliche Leben nicht auf die Dauer behindern, und es werde auch keinen katastrophischen Rückfall in einen vorindustriellen Urzustand geben, vielmehr werde der Mensch die moderne Zivilisation

in aller Ruhe überwinden, langsam und ohne Losreißungsschmerzen, nachdem er von ihr gelernt hat, was zu lernen ihm Not tat; geläutert und gereift wird er aus ihrer Schule hervorgehen. (MSGA 3, 119)

Auch wenn also für Schlick, in direkter Opposition zu den Verfechtern einer protestantischen Ethik im Sinne Webers, Arbeit nicht den Weg ins Paradies wies, brauchte der nach Glückseligkeit strebende Mensch die Hoffnung nicht aufzugeben. Es gab einen anderen, wissenschaftlich begründeten Weg zur Glückseligkeit. 
Einen ersten Hinweis lieferte für Schlick die Beobachtung, dass es Handlungen gab, die nicht der Vorbereitung der Triebbefriedigung dienten, sondern deren Vollzug selbst bereits Triebbefriedigung darstellte. Hierzu zählten Handlungen, die wir im interesselosen und zweckfreien Spiel vollziehen. Der Weg zur vollkommenen Befriedigung unserer Triebe verläuft für Schlick also nicht über die Arbeit, sondern über das Spiel. Spielen, also Handeln, das seinen Zweck in sich selbst findet, macht uns glücklicher als Arbeit, die immer nur das Mittel zum Zweck für etwas anderes ist. Überdies entspricht eine spielerische Existenz besser den eigentlichen Zielen der Evolution, denn, so Schlick

... es kann kein Zweifel sein, daß die Evolution in rastloser Unermüdlichkeit dahin zielt, die Menschen, wie überhaupt alles Lebendige, zu glücklicheren Wesen zu machen. Dies ist seit Darwin der fröhliche Optimismus der Wissenschaft, ... (MSGA 3, 98)

Aus einer evolutionären Perspektive betrachtet sind die Eigenschaften des heutigen Menschen, insbesondere seine Triebstruktur, nicht endgültig festgelegt, sondern veränderlich. „Und nirgends“, prophezeite Schlick, „werden wir am Menschen so interessante Veränderungen wahrnehmen wie gerade in seinen Trieben.“ (98) Gewisse („menschliche, allzu menschliche“) Triebe werden an Kraft verlieren, während andere („übermenschliche“) Triebe an Bedeutung gewinnen werden. Die mit der biologischen Evolution einhergehende Evolution der Triebe garantiert, zumindest „in the long run“, die totale Befriedigung des „Willens zum Glück“. Triebaufschub und temporärer Triebverzicht werden letzten Endes überflüssig, denn

[D]ie Evolution strebt dahin, alle arbeitenden Tätigkeiten allmählich in spielende zu verwandeln. (MSGA 3, 146)

Die Evolution des Menschen wird also in eine spielerische Existenzform münden, in der ein tugendhaftes Leben keiner Anstrengung mehr bedarf. Manche Privilegierte allerdings haben das Glück, diese Existenzform schon heute zumindest in Ansätzen vorwegnehmen zu können. In Schlicks evolutionärer Utopie verwandelt sich das Privileg, das etwa in der antiken Sklavenhaltergesellschaft die Herren gegenüber den Sklaven innehatten, oder im Kapitalismus des 19. Jahrhunderts die Bildungsbürger gegenüber dem Proletariat, in ein „fundamentales Prinzip der Evolution“, so dass in einem späteren Stadium der Evolution alle Angehörigen der Spezies dieses Privileg würden genießen können.

Mit einigen Abstrichen blieben Schlicks Auffassungen über Ethik im Laufe seines gesamten Lebens gleich. Zwar nahm er in Fragen der Ethik (1930, MSGA 
3) den in Lebensweisheit vorherrschenden schwärmerischen Ton zurück und versuchte, seinen Ansatz zumindest oberflächlich an seine später entwickelten logisch-empiristischen Auffassungen von Philosophie anzupassen. Das aber berührte die Grundlagen seiner hedonistischen Ethik kaum. Man sollte sich deshalb nicht von der Tatsache täuschen lassen, dass Schlick in Fragen der Ethik die jugendliche Lebensweisheit verleugnete: Auf Lebensweisheit wird in Fragen der Ethik nirgendwo Bezug genommen. Auch der Nietzsche-Jargon des Frühwerks wird zurückgenommen und die wilden evolutionstheoretischen Spekulationen verschwinden. Der Kern seiner Argumentation bleibt davon unberührt. Moralisches Handeln entspringt aus natürlichen Neigungen, also aus Triebhandlungen. (MSGA 3, 409) Die natürlichen Neigungen des Menschen aber werden durch seine Triebstruktur bestimmt. Die Triebstruktur ist variabel und kann und sollte in Richtung auf eine Verstärkung der „höheren“ sozialen Triebe geändert werden. Die Formung, oder besser die Veränderung der menschlichen Triebstruktur hin zu den „höheren“ Trieben, die er in Lebensweisheit noch dem blinden Wirken der Evolution überlassen hatte, die bestrebt sei, allen Lebewesen ein immer glücklicheres Dasein zu bescheren, wird nun in einem nicht näher spezifizierten Sinne von der Gesellschaft übernommen. Die Gesellschaft belohnt die „sozialen“ Triebe, die somit diejenigen sind, die ihren Trägern am ehesten ein freudenreiches Leben sichern:

Mir ist es nicht zweifelhaft, daß die Erfahrung mit großer Deutlichkeit die sozialen Triebe als diejenigen zeigt, die ihrem Träger am ehesten ein freudenreiches Leben sichern.

Die sozialen Triebe sind diejenigen Dispositionen in einem Wesen, vermöge deren die Vorstellung von freudigen bzw. unlustvollen Zuständen eines anderen Wesens selbst ein lustvolles bzw. unlustvolles Erlebnis ist. (MSGA 3,517 )

Die äußeren Erscheinungsformen der sozialen Triebe sind gütiges Handeln und Altruismus. (MSGA 3, 522). Die hedonistische Ethik ist also, im Gegensatz zur kantischen, die ihren Ausgang nimmt vom Begriff der Pflicht, eine Wunschethik, die mit der Frage beginnt: „Wie muß ich leben, um glücklich zu sein?" Schlicks allgemeine Antwort auf diese Grundfrage lautete, dass aller Wahrscheinlichkeit nach derjenige ein glückliches Leben führen würde, der den sozialen Trieben Priorität vor allen anderen einräumt.

Ein eher zufälliger Unterschied zwischen Lebensweisheit und Fragen der Ethik besteht darin, dass im letzteren das Plädoyer für eine spielerische Existenz, welches in seinem Frühwerk noch einen breiten Raum eingenommen hatte, nicht wieder aufgenommen wird. Das heißt jedoch nicht, dass Schlick diese Thematik 
in seiner Wiener Zeit aufgegeben hätte. Noch 1927 in Der Sinn des Lebens und auch in späteren (unveröffentlichten) Fragmenten verfocht er das Ideal einer „spielerischen Existenz“ des Menschen. An die Stelle des „fundamentalen Prinzips" der evolutionären Verwandlung aller arbeitenden in spielerische Tätigkeiten tritt der Imperativ, jeder solle versuchen, sich eine „spielerische“ Einstellung gegenüber dem Leben zu bewahren, wie sie charakteristisch sei für die „Jugend“, diese nicht verstanden als eine biologische Phase des Lebens, sondern als eine Form der Existenz, die frei von der Herrschaft äußerer Zwecke ihren Sinn in sich selber trage.

Schlicks Mentor Planck hatte für dieses „hinreißende, menschlich ergreifende Lied auf die Jugend“ (so ein enthusiastischer Kritiker) wenig übrig: er bemerkte sarkastisch, er betrachte die Sehnsucht nach dem Glück der Jugend als eine Alterserscheinung. Vielleicht sollte man es sich mit der Verabschiedung von Schlicks Plädoyer für eine mehr spielerische Existenzform nicht ganz so einfach machen. Im Verlauf des 20. Jahrhundert haben eine Reihe ganz unterschiedlicher Denker wie Huizinga, Erikson, Pieper, Russell, Marcuse, oder neuerdings Sennett Vorschläge gemacht, dem Spielerischen oder der Muße (die nicht mit „Freizeit“ oder bloßem Nichtstun zu verwechseln ist) für den Aufbau einer nicht entfremdeten, wirklich menschlichen Gesellschaft mehr Gewicht zu geben. Keiner dieser Autoren hat jedoch Schlicks Arbeiten zur Kenntnis genommen.

Die politische Naivität, wie sie in Lebensweisheit durchscheint, blieb Schlick zeitlebens erhalten. In seiner Wiener Zeit äußerte sie sich z.B. darin, dass er es strikt ablehnte, eine wie auch immer geartete politische Dimension des wissenschaftlichen Wissens auch nur zu diskutieren. Diese Haltung gipfelte in dem Versuch, angesichts der Pressionen der Austrofaschisten jeglichen Kontakt des Vereins Ernst Mach, dessen Vorsitzender er ja war, zur österreichischen Sozialdemokratie zu leugnen und sich stattdessen der Dollfußregierung an den Hals zu werfen, um die Weiterexistenz des Vereins zu sichern, was bekanntlich misslang.

Nach der Habilitation 1910 veröffentlichte Schlick zunächst zahlreiche Rezensionen philosophischer, mathematischer und logischer Werke, bevor er mit der philosophischen Interpretation der Relativitätstheorie ein Thema fand, das ihn in der philosophischen und wissenschaftlichen Szene bekannt machen sollte. Seine erste Publikation in diesem Feld Die philosophische Bedeutung des Relativitätsprinzips datiert von 1915 und kann bis heute als eine hervorragende philosophische Einführung in die Grundgedanken der (speziellen) Relativitätstheorie gelten. Noch wichtiger für Schlicks Reputation aber war Raum und Zeit in der gegenwärtigen Physik (1917), das auch die kurz zuvor von Einstein entwickelte allgemeine Theorie der Relativität berücksichtigte. Der von Fynn Ole Engler und Matthias Neuber herausgegebene Band Über die Reflexion des Lichtes in einer 
inhomogenen Schicht umd Raum und Zeit in der gegenwärtigen Physik (MSGA 2) enthält zwei mustergültig edierte Versionen dieses wichtigen Werkes, einmal die frühe Aufsatzfassung von 1917 und zum anderen die stark erweiterte vierte (und letzte) Auflage der Buchfassung von 1922.

Raum und Zeit wurde schnell eine der erfolgreichsten populärwissenschaftlichen Einführungen in die Grundgedanken der Relativitätstheorie, die einen kaum zu überschätzenden Einfluß auf Generationen von Lesern ausgeübt hat, die mit ganz unterschiedlichen Erwartungen und Voraussetzungen an diesen Text herangetreten sind. Raum und Zeit hat für Philosophen und andere Nichtphysiker das Bild der neuen Theorien geprägt, und umgekehrt hat es vielen Physikern eine bestimmte philosophische Interpretation der Einsteinschen Theorien nahe gelegt.

Die Entstehungsgeschichte von Raum und Zeit ist einigermaßen kompliziert, siehe den editorischen Bericht in Band 2 der MSGA (121-156). Das Werk erschien zuerst 1917 als Aufsatz in den Naturwissenschaften. In der Folgezeit erfuhr es mehrere Neuauflagen und Erweiterungen, 1922 erschien die gegenüber der Aufsatzfassung um mehrere Kapitel erweiterte vierte und letzte Auflage. Die folgende Darstellung orientiert sich an der ersten Auflage, später gehen wir kurz auf die wichtigsten Änderungen in der vierten Auflage ein, die insbesondere die philosophischen Interpretation von Einsteins Theorien betrafen.

Als anschauliche Motivation für den neuartigen Ansatz der speziellen Theorie wählt Schlick Einsteins Erklärung des Michelson-Morley-Experiments. Anstatt wie Lorentz und Fitzgerald das negative Resultat dieses Experiments mithilfe neuer physikalischer Hypothesen („Lorentztransformationen“) zu erklären, beruhte Einsteins Erklärung auf einer tief greifenden begrifflichen Revision unserer grundlegenden Ideen über Raum und Zeit, die in der neuen Theorie „von allen Unklarheiten und unnötigen Denkzutaten befreit [werden] “ (MSGA 2, 305). Um zu erklären, wie diese Revision vonstatten geht, beginnt Schlick mit der fundamentalsten Frage, die man über Zeit und Raum überhaupt stellen kann: "Sind Raum und Zeit eigentlich etwas Wirkliches?" (MSGA 2, 306). Zur Beantwortung dieser Frage folgt Schlick seinem Mentor Planck und zieht das folgende Kriterium heran: „Was man messen kann, das existiert auch.“ (MSGA 2, 307) Mit Poincarés bekanntem Gedankenexperiment der Vergrößerung aller Gegenstände über Nacht zeigt er, dass raumzeitliche Bestimmungen für sich betrachtet Abstraktionen sind, von denen nicht ausgemacht ist, dass ihnen ein realer Sinn zukommt. (MSGA 2, 310) Nur Raum und Zeit zusammen mit den Dingen sind etwas Wirkliches. ${ }^{2}$ In mathematischer Sprache heißt das: „Zwei Welten, die durch eine völlig beliebige (aber stetige und eindeutige)

2. Streng genommen also benutzt er das Wirklichkeitskriterium „Nur was man messen kann, existiert", also die Umkehrung des oben genannten Kriteriums. 
Punkttransformation ineinander übergeführt werden können, sind hinsichtlich ihrer physikalischen Gegenständlichkeit miteinander identisch. (MSGA 2, 312) Anstatt von zwei Welten zu sprechen erweist es sich als zweckmäßiger, von zwei funktional äquivalenten Koordinatensystemen zu sprechen, die die eine Welt nur verschieden beschreiben. Ein Koordinatensystem aber ist nichts anderes als eine Geometrie. Von der Geometrie eines Raumes, und das heißt auch, von empirisch sinnvollen Messungen räumlicher und zeitlicher Größen kann man also nur sprechen, wenn man das physikalische Verhalten der räumlichen Körper in die Betrachtung einbezieht, was schon Poincaré so formulierte: „Der Raum ist in Wirklichkeit gestaltlos, und allein die Dinge, die darin sind, geben ihm eine Form." (MSGA 2, 316) Die allgemeine Relativitätstheorie führt dann zu der folgenden präzisierenden Verallgemeinerung dieser These:

Raum und Zeit sind nur in der Abstraktion von den physischen Dingen und Vorgängen trennbar. Wirklich ist nur die Vereinigung, die Einheit von Raum, Zeit und Dingen; jedes für sich ist eine Abstraktion. Und bei einer Abstraktion muß man sich immer fragen, ob sie auch naturwissenschaftlichen Sinn hat, d.h. ob das durch die Abstraktion Getrennte auch tatsächlich von einander unabhängig ist. (MSGA 2, 320)

Diese Einsicht ermöglichte es Einstein, so Schlick, den fruchtlosen Streit zwischen Anhängern und Gegnern der Existenz absoluter (beschleunigter) Bewegungen auf eine neue Ebene zu heben. Einerseits sei gegen Newton zu sagen, dass es keine zureichenden Gründe für die Annahme der Existenz solcher Bewegungen gibt, andererseits reichen Machs allgemeine Argumente für die Relativität aller beschleunigten Bewegungen nicht aus. Vom Standpunkt der Erfahrung gebe es für keine der beiden Positionen zwingende Argumente. Ein entscheidendes Verdienst von Einsteins allgemeiner Theorie bestehe nun darin, ein gutes empirisches Argument für die allgemeine Relativität auch beschleunigter Bewegungen gefunden zu haben, nämlich die Identität von träger und schwerer Masse. (MSGA 2, 326) Danach kann die beobachtbare Beschleunigung eines Körpers an irgendeinem Punkt des Universums entweder als Trägheits- oder als Gravitationswirkung aufgefasst werden. Dies hat drastische mathematische Konsequenzen. Wenn man das allgemeine Relativitätspostulat aufrecht erhalten will, wonach es keine ausgezeichneten Bezugssysteme gibt, muß man darauf verzichten, die raumzeitlichen Beziehungen von Körpern mit Hilfe euklidischer Methoden zu beschreiben. Der Raum oder besser die Raumzeit hat nur noch die Struktur einer allgemeinen Riemannschen Mannigfaltigkeit mit variabler Riemannscher Metrik, die nur „infinitesimal“ euklidisch ist. 
Raum und Zeit für sich sind niemals Gegenstände der Messung; sie bilden zusammen nur ein vierdimensionales Schema, in welches wir mit Hilfe unserer Beobachtungen und Messungen die physikalischen Objekte und Prozesse einordnen (MSGA 2, 331).

Diese Einordnung folgt einem Stetigkeitsprinzip, dem zufolge für kleine Änderungen die Einordnung der allgemeinen Relativitätstheorie die Messungen der gewöhnlichen Newtonschen Physik approximiert („eine Art Kontinuitätsprinzip“). (MSGA 2, 337)

Wie im editorischen Bericht der Herausgeber detailliert ausgeführt wird, erfährt Raum und Zeit in den folgenden Auflagen erhebliche Umarbeitungen und Erweiterungen. So kommen einige vollständig neue Kapitel hinzu. Insbesondere setzte sich Schlick in einem neuen Schlusskapitel „Beziehungen zur Philosophie“ mit Argumenten Cassirers auseinander, die dieser in seinem 1921 erschienenen Buch Zur Einsteinschen Relativitätstheorie zugunsten einer neukantianischen Interpretation von Einsteins Ergebnissen vorgebracht hatte.

Raum und Zeit war ein äußerst erfolgreiches Buch, das Schlicks Ruf als eines führenden philosophischen Interpreten der Relativitätstheorie begründete. Auch wenn Schlick zunächst noch nicht zu einer streng empiristischen Interpretation der Relativitätstheorie gelangte, wendete er sich doch von Anfang an gegen Versuche neukantianischer Philosophen wie Natorp und Cassirer, die Relativitätstheorie als Beleg für die Richtigkeit der neukantianischen Wissenschaftsphilosophie zu vereinnahmen. Die Logischen Empiristen selbst, aber auch spätere Wissenschaftsphilosophen wie Coffa behaupteten, Schlicks philosophische Interpretation der Relativitätstheorie und seine Kritik an Cassirers Versuch, Einsteins Theorie als mit der neukantianischen Philosophie kompatibel zu erweisen, hätten endgültig gezeigt, dass die neukantianische Wissenschaftsphilosophie mit der Naturwissenschaft des 20. Jahrhunderts unvereinbar sei. Die Plausibilität dieser bündigen Bestandsaufnahme ist neuerdings in Zweifel gezogen worden, etwa von Ryckman in The Reign of Relativity (Ryckman 2005) und in einigen Arbeiten Friedmans. In der Tat gibt es einige triftige Argumente dafür, dass Schlick in der Debatte um die Rolle des synthetischen Apriori in der empirischen Erkenntnis Cassirers Ansatz, der von einem synthetischen Apriori ausging, das einen genetischen Charakter besaß und eine historische Entwicklung durchlief, missverstanden hat. Auch wenn dies so ist, ändert das nichts an der Tatsache, dass Schlicks empiristische Interpretation der Einsteinschen Theorien für lange Zeit so etwas wie die herrschende Meinung war („the only game in town“), und der Neukantianismus als Wissenschaftsphilosophie von den Wissenschaftlern selbst (bis auf wenige Ausnahmen wie Weyl oder Margenau) kaum zur Kenntnis, geschweige denn ernst genommen wurde. 
Machen wir nun einen weniger sachlich als rezensionstechnisch begründeten Sprung zu Schlicks Spätwerk, d.h. den Werken, die er in seinem letzten Lebensjahrzehnt (1926-1936) in Wien verfasst hat. Band 6 der Kritischen Gesamtausgabe, detailliert und kenntnisreich herausgegeben, eingeleitet und kommentiert von Johannes Friedl und Heiner Rutte, enthält die meisten dieser kleineren Arbeiten, die zwischen 1926 und 1936 erschienen sind. Nicht aufgenommen wurden einige Texte wie Form and Content und L'école de Vienne et la Philosophie traditionelle, die zwar im genannten Zeitraum entstanden sind, aber erst postum veröffentlicht wurden. Für alle, die an Schlicks Philosophie nicht nur in großen Zügen, sondern auch in den Details interessiert sind, an ihren internen Verschiebungen und subtil veränderten Schwerpunktsetzungen, ist die Lektüre dieser opera minora ein Muß. Sehr hilfreich dafür sind die Einführungen, Kommentare und Verweisungen der Herausgeber, die die Entstehungsgeschichte, den philosophischen Kontext und das wissenschaftliche Umfeld beleuchten, in denen diese Arbeiten entstanden sind.

Wie alle bisher erschienenen Bände der kritischen Gesamtausgabe ist auch dieser von herausragender editorischer Qualität. Der Band enthält insgesamt 40 Texte, die sich in zwei Kategorien einteilen lassen: zum einen 16 kurze Rezensionen und kleinere akademische Gelegenheitsarbeiten, zum anderen 24 Artikel, die in philosophischen Zeitschriften und Kongressberichten erschienen sind.

Die in (MSGA 6) gesammelten Rezensionen sind kurze, für ein allgemeines philosophisches Publikum geschriebene Texte, die meist wohlwollend, aber philosophisch nicht in die Tiefe gehend über den Inhalt der zu besprechenden Werke berichten. Unter den besprochenen Autoren finden sich neben heute vergessenen Autoren unter anderen auch Schlicks philosophische Diskussionspartner Carnap, Russell, Reichenbach und Bridgman.

Philosophisch bedeutsamer sind die (MSGA 6) versammelten Aufsätze. Neben „Klassikern“ wie Erleben, Erkennen, Metaphysik (1932), Die Wende der Philosophie (1932) oder Das Fundament der Erkenntnis (1934), hat Schlick während seiner Wiener Zeit auch eher „abseitige“ Arbeiten verfasst wie Der Sinn des Lebens (1927), die mit dem Logischen Empirismus des Wiener Kreises höchstens indirekt in Verbindung stehen und eher als Reprise seiner Jugendwerke aufzufassen sind. Außerdem enthält der vorliegende Band eine Reihe englischsprachiger Arbeiten Schlicks, die bislang so gut wie unzugänglich waren, und die Schlick im Rahmen seiner Verpflichtungen als Gastprofessor in Berkeley verfasste. Dazu kommen einige Aufsätze in französischer Sprache, die sich aus seinen Verbindungen mit Vertretern der „wissenschaftlichen Philosophie“ in Frankreich ergaben. Schlick kann, das dokumentieren die in diesem Band versammelten Arbeiten, wohl als einer der ersten „internationalen“ Philosophen des 20. Jahrhunderts angesehen werden. 
Die große Zahl der hier versammelten Texte, die ein weites Feld von Themen und Problemen abdecken, lassen es nicht ratsam erscheinen, sie in dieser Besprechung alle im Einzelnen zu behandeln. Stattdessen möchte ich von der Freiheit des Rezensenten Gebrauch machen und nur auf einige Texte genauer eingehen, die mir aus verschiedenen Gründen besonders bemerkenswert erscheinen, sei es, weil sie zu den kanonischen Texten gehören, die man von Schlick gelesen haben sollte, sei es, weil sie Facetten von Schlicks philosophischer Persönlichkeit beleuchten, die oft zu wenig beachtet werden.

Es wäre zu einfach, die Jahre 1922-1936 lediglich als Schlicks „logischempiristische Periode " zu charakterisieren. Schlick bleibt in Bezug auf den Kreis, dessen Mittelpunkt er bildete, durchaus „exzentrisch“, wie etwa sein anhaltendes Interesse an ethischen und philosophiehistorischen Fragen belegt. Das wird ersichtlich nicht nur aus seinen in der Wiener Zeit entstandenen Schriften, sondern auch aus seinen Lehrveranstaltungen. Das Verzeichnis der von Schlick in Wien gehaltenen Seminare und Vorlesungen hat sich erhalten und zeigt ein recht breites Themenspektrum, das weit über die Wissenschaftstheorie hinaus reichte. Es finden sich Veranstaltungen zur Grundlegung der Metaphysik der Sitten, zu Schopenhauer und sogar zu Hegel. Wiederholt behandelte er Russells nichttechnische Werke Probleme der Philosophie oder Einführung in die mathematische Philosophie. Dazu kamen aber auch allgemeine Themen wie Philosophie der Psychologie, der Wahrscheinlichkeit, oder Gestalttheorie. Im Vorlesungsverzeichnis einer deutschsprachigen Universität unserer Tage würde Schlicks Veranstaltungsliste kaum als antiquiert auffallen.

Auch die wissenschaftsphilosophischen Arbeiten im engeren Sinne bilden keineswegs eine homogene Klasse. Einige verraten deutlich den Einfluß Wittgensteins, andere verwenden Argumente aus der Philosophiegeschichte, was bei anderen Mitgliedern des Kreises eher unüblich war. Eine Gruppe für sich bilden die im engeren Sinne logisch-empiristischen Arbeiten, die sich insbesondere auf die Protokollsatzdebatte beziehen, in der zum ersten Mal kreisinterne Gegensätze öffentlich diskutiert wurden.

Mit Schlicks Wechsel nach Wien begann also keineswegs sofort eine ganz neue, eben die logisch-empiristische Periode seines Philosophierens. Ältere Strata seines Denkens blieben durchaus noch für längere Zeit sichtbar und wurden erst langsam von neueren Komponenten überlagert oder verdrängt. Manches erhielt sich praktisch unverändert als Zeuge längst vergangener Denkepochen. Dazu gehört zum Beispiel der Aufsatz Der Sinn des Lebens (1927), der als eine Art Fossil die Themen und Denkweisen aus seiner frühesten Zeit, insbesondere aus seinem Jugendwerk Lebensweisheit. Versuch einer Glückseligkeitslehre (1908) fast unverändert wieder aufnimmt, als hätte es die Begegnung mit Wittgenstein und dem Wiener Kreis nie gegeben. 
Die philosophisch interessanten Veränderungen, die sich während der Wiener Jahre in Schlicks Denken ereigneten, betrafen wie gesagt nicht die Ethik, sondern ereigneten sich auf anderen Gebieten. Sie führten ihn insbesondere von einem strukturellen Realismus, wie er ihn in der Allgemeinen Erkenntnislehre vertreten hatte, zu seiner Version des logischen Empirismus, die bis heute nicht in allen Einzelheiten aufgearbeitet und verstanden worden ist. Immerhin aber kann man sagen, dass sein logischer Empirismus keineswegs identisch war mit dem Carnaps oder gar Neuraths. Einen guten Beleg bietet dafür der Aufsatz Erleben, Erkennen, Metaphysik (1926). Zwar knüpfte er weiterhin an die in der Allgemeinen Erkenntnislehre entwickelte Unterscheidung von „Erkennen“ und „Kennen (Erleben)“ an, jedoch radikalisierte er sie zu der These, erkennen könne man nur die „reine Form“ oder „Struktur“ der Welt, während alle erlebten qualitativen Bewusstseinsinhalte grundsätzlich „privatim“ und noch nicht einmal sprachlich mitteilbar seien (MSGA 6, 38).

Alle unsere Aussagen, mögen sie sich nun auf die gewöhnlichen Tatsachen der Lebenswelt beziehen oder wissenschaftliche Tatsachen betreffen, gäben immer nur formale Beziehungen der Welt wieder. Jede scheinbar noch so „anschauliche“ Erkenntnis war deshalb nach Schlick genauso „abstrakt“ wie die Erkenntnis der Physik. Diese Auffassung führte ihn dann dazu, den Unterschied zwischen „Realismus“ und „Positivismus“ einzuebnen: für die strukturelle Konzeption von Erkenntnis ist es gleichgültig, ob man den Gegenständen realistisch eine „selbständige Wirklichkeit“ zuschreibt oder diese positivistisch als „gedankliche Konstruktionen“ auffasste (MSGA 6, 42, 43), da in die Erkenntnis ja nur ihre Struktur eingeht. Das ist eine typisch logisch-positivistische Auffassung, die sich zum Beispiel auch im Aufbau findet. Noch stärker akzentuiert wurde der neue logisch-empiristische Standpunkt in Positivismus und Realismus (1932), wo Schlick auf die antipositivistischen Überzeugungen prominenter Physiker wie Einstein, Planck und Sommerfeld reagierte. Dies führte zu einer gewissen Annäherung an Mach, dessen Phänomenalismus er in der Allgemeinen Erkenntnislehre noch vehement abgelehnt hatte.

Am deutlichsten markiert vielleicht Die Wende in der Philosophie (1930) Schlicks Adaption an die Wiener Umgebung. Schon bei oberflächlicher Lektüre verrät der Text den massiven Einfluß Wittgensteins. Nach Schlick verdanken wir Wittgensteins Tractatus die grundlegende neue Einsicht in das Wesen der Philosophie. Danach besteht ihr Zweck nicht in der Aufstellung genuin philosophischer Sätze, sondern in der logischen Klärung der Gedanken: „Die Philosophie ist keine Lehre, sondern eine Tätigkeit.“ (Tractatus 4.112). Das führte zu einer akkuraten Arbeitsteilung: die Wissenschaften verifizieren Sätze, die Philosophie klärt den Sinn wissenschaftlicher Aussagen. Die Wende kann zunächst als Gegenentwurf zum „offiziellen Programm“des Wiener Kreises 
gesehen werden, dem so genannten Manifest des Wiener Kreises, das der „linke Flügel“ des Kreises 1929 veröffentlicht hatte und das Schlick höchlich missfallen hatte. Neben Wittgensteins Einfluß lassen sich in der Wende auch stilistische Einflüsse aus früheren Phasen seines Denken konstatieren, etwa wenn Schlick die Philosophie als Königin der Wissenschaften apostrophiert, die zwar selbst keine Wissenschaft ist, aber ihnen „Inhalt, Seele und Geist“ verleiht und so „das Alpha und Omega aller wissenschaftlichen Erkenntnis ist." In gewissem Sinne explizierte Schlick in Wende das Modell von Philosophie, das er schon in Raum und Zeit praktiziert hatte, nämlich die Philosophie als Explikation der Naturwissenschaften.

Eine Fortsetzung und Adaption der Thematik der Wende an die Bedürfnisse eines englischsprachigen Publikums finden sich in den beiden Arbeiten The Future of Philosophy (1931) und The Future of Philosophy (1934). Dort begründete er die neue Konzeption der Philosophie als einer Tätigkeit nicht nur sprachlogisch, sondern auch durch Rekurs auf die Philosophiegeschichte, indem er Spuren des neuen Verständnisses von Philosophie schon bei Sokrates, Leibniz und Kant aufzuweisen versucht. $(376,387)$ Diesen Ansatz führte er, was später noch genauer behandelt werden soll, ins Extrem, wenn er in dem postum erschienenen Aufsatz L'école de Vienne et la Philosophie traditionelle (1938) Sokrates als eigentlichen Lehrer des Wiener Kreises identifizierte.

Über das Fundament der Erkenntnis (1934) war ein im Kreis höchst umstrittener Beitrag zur Protokollsatzdebatte. Ob Schlick als erkenntnistheoretischer „Fundamentalist“ anzusehen sei, ist unter Experten durchaus umstritten. Dass diese Frage keineswegs so einfach zu beantworten ist, wie man meinen könnte, wird schon daraus ersichtlich, dass nach Schlick „Konstatierungen“ nicht als Fundament der Erkenntnis im üblichen Sinne aufzufassen waren:

[Konstatierungen] ... liegen keineswegs am Grunde der Wissenschaft, sondern die Erkenntnis züngelt gleichsam zu ihnen auf, jeden nur in einem Augenblick erreichend und ihn sogleich verzehrend. Und neu genährt und gestärkt flammt sie dann zum nächsten empor. Diese Augenblicke der Erfüllung und des Verbrennens sind das Wesentliche. Von ihnen geht alles Licht der Erkenntnis aus. Und dies Licht ist es eigentlich, nach dessen Ursprung der Philosoph fragt, wenn er das Fundament des Wissens sucht." (MSGA 6, 514)

Für den wenig feinsinnigen Neurath dokumentierten diese Ausführungen einen schweren Fall von Begriffslyrik, was der empfindliche Schlick sehr übel nahm, wie er Carnap in einem Brief kundtat. Deutlich brachte er seine Kritik am seiner Meinung nach zu rationalistischen Positivismus Neuraths und Carnaps in Facts 
and Propositions (1935) zur Sprache. In diesem Aufsatz versuchte er seine These, wonach Sätze durchaus mit Tatsachen vergleichbar seien, als Standpunkt eines bodenständigen Empiristen gegen die rationalistischen Übertreibungen seiner "guten Freunde und Gegenspieler“ (MSGA 6, 573) in der Protokollsatzdebatte darzustellen. Konstatierungen fungierten für ihn dabei als letzte, nicht weiter diskutierbare Garanten für die Wahrheit wissenschaftlicher Sätze. In Sur les constatations (1935) hält Schlick an dieser Konzeption fest, versucht sie aber diesmal durch ein Wittgensteinsches Argument zu stützen, wonach sich „die Grammatik der Konstatierungen“ wesentlich von „der Grammatik der Protokollsätze“ unterscheide. (MSGA 6, 664) Was den Status von Konstatierungen angeht, scheint es unter den Mitgliedern des Kreises nicht mehr zu einer Einigung gekommen zu sein. Die Protokollsatzdebatte endete, ohne dass sich die Kontrahenten darüber geeinigt hätten, was unter Protokollsätzen oder Konstatierungen zu verstehen sei.

Der Aufsatz Gibt es ein materiales Apriori? (1932) nimmt eine Thematik wieder auf, die Schlick schon seit den Anfängen seiner philosophischen Laufbahn beschäftigt hatte, nämlich die Rolle der Anschauung in der Erkenntnis. In scharfer Opposition zur Phänomenologie Husserls und Schelers verteidigte er die schon in der Allgemeinen Erkenntnislehre vertretene These, dass es nur ein formales Apriori geben könne, eine Einsicht, die, wenn auch unvollkommen, bereits in Kants Konzeption dieses Begriffs angelegt gewesen sei. Versuche der Phänomenologen, das Kantische synthetische Apriori auf Farbinkompatibilitäten und ähnliche Phänomene auszuweiten, lehnt er als unkantisch und sachlich unhaltbar strikt ab. Mit Wittgenstein argumentiert er, vermeintlich synthetisch apriorische Sätze wie „Nichts ist zugleich rot und grün“ oder „Ein Körper mit dem Gewicht $2 \mathrm{~kg}$ kann nicht $3 \mathrm{~kg}$ wiegen“ ergäben sich analytisch aus der Grammatik der Farben oder des Gewichtsbegriffs. Tatsächlich ist die Beziehung zwischen Phänomenologie und Wittgensteinscher Grammatik nicht so einfach, wie Schlick sie darstellt. Jaakko und Meryll Hintikka etwa haben in Investigating Wittgenstein argumentiert, man könne, zumindest für eine gewisse Zeit, durchaus von einer Wittgensteinschen Phänomenologie sprechen.

Eine elegante Kritik der damals verbreiteten „Ganzheitsphilosophie“ findet sich in dem kleinen Aufsatz Über den Begriff der Ganzheit (1935). Schlick gibt darin ein Musterbeispiel einer elementaren logischen Analyse eines bei damaligen „traditionellen“ Philosophen sehr geschätzten opaken Begriffs, dessen unkritische Verwendung zu überflüssigen Unklarheiten und Scheinproblemen führt. Üblicherweise wurde der Begriff der „Ganzheit“ (oder „Gestalt“) dem Begriff der bloßen "Summe“ entgegengesetzt, wobei man davon ausging, dass der Begriff „Summe“ philosophisch unproblematisch sei. In diesem Verständnis ist dann eine Melodie mehr als die Summe ihrer Töne und ein Organismus ist mehr 
als die Summe der Zellen, aus denen er besteht. Schlick weist darauf hin, dass man sich auf diese Weise die Dinge etwas zu einfach macht. Es ist z.B zunächst durchaus nicht klar, was unter einer Summe von Geschwindigkeiten oder einer Summe von Temperaturen zu verstehen ist. Eine Summe physikalischer Größen ebenso wie eine Ganzheit muß vielmehr definiert werden. Wie das geschieht, ist erst einmal willkürlich. Deswegen bezeichnen die Worte „ganzheitlich“ und „summenhaft“ nicht verschiedene objektive Eigenschaften irgendwelcher Gebilde, sondern sie bedeuten zunächst verschiedene Darstellungsarten. (MSGA 6, 556) Die „ganzheitliche“ Philosophie glaubt sich der begrifflichen Arbeit einer Definition von Ganzheiten enthoben und vermeint, einen direkten „intuitiven“ Zugang zu der Sphäre der „Ganzheiten“ zu haben, die der empirischen Forschung weitgehend entzogen ist. Das führt, so Schlick, in die Untiefen der Metaphysik.

Eine populärwissenschaftlich gehaltene Anwendung der in Wende skizzierten Konzeption von Philosophie als Tätigkeit auf das Problem ihrer Beziehung zu den Geistes- und Naturwissenschaften findet sich in Philosophie und Naturwissenschaft (1934). Nach Schlick weist die übliche Vorstellung des Verhältnisses zwischen Philosophie und Naturwissenschaften der Philosophie eine systematisierende oder einheitsstiftende Rolle zu: Die Naturwissenschaften entwerfen ein „Weltbild“ der Wirklichkeit, was die Philosophie zur Herstellung einer „Weltanschauung“ benutzen (MSGA 6, 522). ${ }^{3}$ Schlick lehnte diese Auffassung nicht rundweg ab, hielt sie aber in wesentlichen Punkten für revisionsbedürftig. Nach ihm ist die Philosophie überhaupt keine Wissenschaft, geschweige denn eine Grundwissenschaft, sondern eine Tätigkeit, „durch welche der Sinn aller zur Erkenntnis notwendigen Begriffe erklärt wird.“ (MSGA 6, 527) Das Verhältnis zwischen Naturwissenschaft und Philosophie beschreibt Schlick vage als den „eigentümliche(n) Prozeß, durch den die wahre Bedeutung der naturwissenschaftlichen Begriffe entdeckt, und von ihnen ausgehend die Weltanschauung geformt wird." (MSGA 6, 528)

Die Naturwissenschaften aber, besser gesagt ihre Grundbegriffe, bestimmen die wesentlichen Züge der Weltanschauung. Die anderen Wissenschaften, insbesondere die Geisteswissenschaften spielen eine sekundäre „bewertende“ Rolle. Dabei verwenden sie im Wesentlichen dieselben Begriffe wie die, die „schon im Leben und Treiben des Alltags gebraucht werden." (MSGA 6, 537) Die Naturwissenschaften sind begrifflich weiter entwickelt und deshalb, so Schlick, „philosophischer“ als die Geisteswissenschaften. Philosophie und Naturwissenschaft ist auch insofern bemerkenswert, als er eine der wenigen nichtpolemischen

3. Wie weit in Schlicks Verwendung des Ausdrucks „Weltanschauung“ eine unterschwellige Polemik gegen Neurath mitschwingt, der den Begriff, Weltanschauung“ vehement ablehnte und stattdessen immer von „wissenschaftlicher Weltauffassung“ sprach, muß hier offenbleiben. 
Darstellungen der Positionen des südwestdeutschen Neukantianismus (Rickert und Windelband) enthält, die jemals von einem Mitglied des Wiener Kreises verfasst worden ist. ${ }^{4}$

Die Arbeit Meaning and Verification (1936) geht auf eine Anregung von Clarence Irving Lewis zurück, etwas für eine bessere Verständigung zwischen amerikanischem Pragmatismus und logischem Empirismus des Wiener Kreises zu tun. Zu diesem Zweck hatte Lewis seinen 1934 erschienenen Essay Experience and Meaning an Schlick und Carnap geschickt und angeregt, das Projekt eines Vergleichs zwischen beiden Schulen ins Auge zu fassen. Meaning and Verification war Schlicks Antwort auf Lewis, in der er den Wiener logischen Positivismus gegen Lewis' pragmatistische Kritik verteidigte. Insbesondere versucht Schlick Lewis' Einwand zu widerlegen, der Wiener Ansatz sei einem „methodologischem Solipsismus“ verpflichtet, der empirisches Wissen grundsätzlich als Wissen eines einsamen Subjektes konzipiere. Mit Lewis stimmt er darin überein, dass ein wissenschaftlicher Empirismus (wie ihn sowohl der Wiener Kreis wie auch der amerikanische Pragmatismus anstrebten) durchaus nicht auf einen Solipsismus festgelegt sei. Gegen Lewis versucht er zu zeigen, dass nur eine oberflächliche Interpretation etwa von Carnap's Aufbau, die Meinung nahelegen könne, die Wiener Empiristen verträten eine solche Auffassung. Meaning and Verification kann als Beginn einer letztlich wenig ertragreichen Diskussion zwischen amerikanischen Pragmatisten und logischen Empiristen verstanden werden, die sich bis in die sechziger Jahre hinein fortsetzte, aber kaum konkrete Ergebnisse zeitigte. Ein Grund dafür mag gewesen sein, dass im Gegensatz zu Schlick Carnap, den Lewis ebenfalls angesprochen hatte, recht wenig Interesse an einem ernsthaften Dialog mit Lewis, Morris, Dewey und anderen amerikanischen Pragmatisten zeigte.

In Stil und Duktus war Schlick ein bürgerlicher Philosoph des langen 19. Jahrhunderts mit leicht bohemehaften Zügen, wie man bereits an seinem etwas exaltiert formulierten Hedonismus der Lebensweisheit sehen kann, was aber auch in vielen seiner späteren Äußerungen durchscheint. Schlick besaß eine Weltläufigkeit, die den meisten anderen Mitgliedern des Kreises, zumindest am Anfang ihrer Karriere, abging. Er leistete einen wesentlichen Beitrag zur Internationalisierung des Kreises, insbesondere durch seine Kontakte in die USA sowie nach England und Frankreich. Schlicks großbürgerlich-aristokratischer Lebensstil hatte auch Auswirkungen auf den Stil seines Philosophierens. In der Protokollsatzdebatte agierte er längst nicht so verbissen wie manche seiner

4. Während Schlick in der ursprünglichen Fassung von Philosophie und Naturwissenschaften noch von „besonderen Verdiensten“ Rickerts und Windelbands für die Wissenschaftsphilosophie der Geisteswissenschaften sprach, ist allerdings in der in Erkenntnis veröffentlichten Version nur noch von "gewissen Verdiensten“ die Rede. 
Gegenspieler. Eine spielerische Nonchalance in seinem Philosophieren, die man auch als Arroganz interpretieren konnte, ist manchmal kaum zu überhören:

When in the spring of last year I wrote my short paper on Das Fundament der Erkenntnis sitting leisurely on a balcony overlooking the blue bay of Salerno ... (MSGA 6, 567).

Bei manchen Gelegenheiten legte er ein geradezu divenhaftes Verhalten an den Tag, etwa wenn er gegenüber Carnap brieflich seine "Verwunderung" über Neuraths drastische Kritik am Fundament der Erkenntnis zum Ausdruck brachte und anschließend bemerkte: „Ich werde natürlich nicht direkt darauf antworten, sondern höchstens gelegentlich einmal etwas schreiben, was man als Antwort auffassen kann, wenn man will." (MSGA 6, 562) Anekdoten wie diese belegen, dass die Kommunikation zwischen den Mitgliedern des Kreises keineswegs immer den Normen eines rationalen, rein am Inhaltlichen interessierten Diskurses folgte, wie ihn Carnap idealisierend in seiner Intellektuellen Autobiographie beschrieb, sondern wesentlich wohl auch durch die sozial und klassenspezifisch bestimmten Verhaltenseigenarten der Beteiligten bestimmt wurde.

Wie weit sich Schlick offenbar vom mainstream des logischen Empirismus in seinen letzten Jahren entfernt hatte, wird ersichtlich aus einer seiner letzten Schriften, seinem Beitrag L'école de Vienne et la philosophie traditionelle zum Internationalen Descartes Kongress 1937 in Paris, der erst postum erschienen ist. $^{5}$ Darin geht er so weit, ethische und moralische Fragen als das eigentliche Zentrum der philosophischen Tätigkeit der „Wiener Schule“ zu deklarieren:

Der „wahre Vater unserer Philosophie“ ist weder ein Gelehrter noch ein Logiker, weder Comte, noch Frege, noch Poincaré, noch Russell, ... sondern Sokrates. Er war der erste, der seine Schüler die Kunst lehrte, richtige Fragen zu stellen.

In Wirklichkeit verhält sich die,Wiener Schule der Philosophie' zu Fragen der Werte und der Moral genauso wie die Philosophie des Sokrates: für sie ist die Ethik eine Aufgabe der Philosophie und sie weiß, daß die Klärung der moralischen Begriffe unendlich wichtiger für die Menschen ist als alle theoretischen Probleme.

Für den späten Schlick war also die „Wiener Schule der Philosophie“ nur eine „Vorschule" der Ethik, die zum Bedauern ihres Oberhauptes noch nicht zu ihrem

5. Als zu Lebzeiten nicht veröffentlichter Text soll diese Arbeit in einem späteren Band der MSGA erscheinen. 
eigentlichen Thema, eben der Klärung moralischer Begriffe, vorgedrungen war. Es ist nicht klar, wie viel von dieser gelinde gesagt neuartigen Interpretation des Wiener Kreises dem Versuch einer Anpassung an den Kontext des damals bereits in Österreich herrschenden Austrofaschismus geschuldet war, sicher ist, dass sie logischen Empiristen wie Neurath, Carnap oder Frank nicht gefallen hat. Sie belegt einmal mehr die exzentrische Position Schlicks innerhalb des Wiener Kreises. Diese Exzentrizität verträgt sich zwar gut mit seinen philosophischen Anfängen, also der Lebensweisheit, aber kaum mit dem aufklärungsorientierten, engagierten wissenschaftlichen Modernismus, wie er etwa im Manifest oder im Vorwort des Aufbaus formuliert wurde.

Vielleicht kann man L'école de Vienne tatsächlich als genuinen Ausdruck von Schlicks bürgerlichem Logischen Empirismus lesen. Das wäre nicht neu, schon Feigl hatte in seinem etwas hymnischen Nachruf Schlicks Philosophieverständnis als „sokratische Tätigkeit der Sinnerklärung“ charakterisiert. Dieser Empirismus lehnte Carnaps Konzeption von Philosophie als Wissenschaftslogik explizit ab und hatte erst recht nichts übrig für Neuraths Projekt, Philosophie durch „Einheitswissenschaft“ zu ersetzen. Stattdessen sah er sich als Statthalter der einen philosophia perennis, die seit jeher im Kampf mit den schnell wechselnden „Modephilosophien “verschiedener Provenienz lag. Spezifische Probleme des modernen Wissens und der modernen Gesellschaft gerieten dabei nicht mehr ins Blickfeld. Für Schlicks Wissenschaftsphilosophie bedeutete das insbesondere, dass in ihr die Rolle von Wissenschaft und Technik in der modernen Industriegesellschaft nicht thematisiert wurde. Stattdessen bettet sich seine Spätphilosophie ein in eine vage, bildungsbürgerlich konzipierte Aufklärung, die die großen Namen der abendländischen Geistesgeschichte Revue passieren läßt, die aber für wesentliche Aspekte einer modernen Gesellschaft des 20. Jahrhunderts blind blieb.

Außerhalb der Wissenschaftsphilosophie war Schlick kein moderner Denker. Vielleicht könnte man ihn als einen aufgeklärten Vertreter der Mandarintradition des deutschen Gelehrtentums ${ }^{6}$ charakterisieren, die spätestens mit der Weimarer Republik und der Ersten Republik in Österreich unterging. Eine solche Einschätzung zu geben, heißt nicht Schlicks moderaten bürgerlichen Logischen Empirismus gering zu schätzen. Um das einzusehen, genügt es, sich vor Augen zu halten, was für eine Art von Philosophie in den folgenden Jahrzehnten an der Universität Wien - weit über das Ende des tausendjährigen Reiches hinaus - den Ton angeben sollte.

Schlicks philosophisches Werk ist unvollendet geblieben. Ein opus magnum hat er nach der Allgemeinen Erkenntnislehre (1918/1925) nicht mehr geschrieben, und die kleineren Arbeiten, die er in seinen Wiener Jahren verfasste, können

6. Vgl. Fritz Ringer 1969: The Decline of the German Mandarins. Cambridge Mass.: Harvard University Press. 
kaum als Fragmente eines größeren Werkes gesehen werden, das sich später vielleicht aus ihnen ergeben hätte. Sie bleiben membra disiecta eines moderaten, in wesentlichen Zügen an das 19. Jahrhundert erinnernden, logischen Empirismus, der versuchte, Weisheit und Wissenschaft zusammen zu denken. Auch wenn ihm das aus heutiger Sicht wohl nicht völlig überzeugend gelungen ist, markiert Schlicks Werk einen Meilenstein in der Entwicklung der Wissenschaftsphilosophie. Die jetzt im Erscheinen begriffene, in wichtigen Teilen bereits vorliegende Gesamtausgabe eröffnet optimale Möglichkeiten, sich Schlicks philosophisches Erbe kritisch anzueignen. ${ }^{7}$

7. Ich danke Anne Siegetsleitner und Massimo Ferrari, dass sie mir einige ihrer (unveröffentlichten) Arbeiten zu Schlicks Philosophie zur Verfügung gestellt haben. 